\title{
Visualization of water droplet deformation and breakup in a continuously accelerated flow field.
}

\author{
A. García-Magariño* ${ }^{1}$, S. Sor ${ }^{2}$, A. Velazquez ${ }^{3}$ \\ ${ }^{1}$ Engineering Systems for Spanish Defense (ISDEFE) Madrid, Spain \\ ${ }^{2}$ National Institute of Aerospace Technology Madrid, Spain \\ ${ }^{3}$ Universidad Politécnica de Madrid, Madrid, Spain \\ *Corresponding author: agarcia-magarino@isdefe.es
}

\begin{abstract}
A new specific direct illumination technique is proposed for studying the deformation and breakup process of droplets that are exposed to a continuously increasing flow field. This type of flow field is in contrast to the traditional shock-tube experiments where droplets are suddenly exposed to a constant high airstream. In order to generate a continuously accelerated flow field the rotating arm facility at INTA is used. Droplets are allowed to fall in the path of an incoming airfoil mounted at the end of a rotating arm. Under certain conditions, these droplet deform and breakup before impinging on the airfoil. The incoming airfoil is a key element in the illumination technique. Droplets of $0.8 \mathrm{~mm}$ of diameter immersed in the flow field generated by an airfoil model of chord $0.690 \mathrm{~m}$ approaching at $80 \mathrm{~m} / \mathrm{s}$ were chosen for this study and were found to undergo a breakup process similar to the bag and stamen breakup found in the literature. Each of the stages at this type of breakup process was investigated by means of this new direct illumination technique and a high resolution camera. These images were compared to those images obtained using shadowgraph illumination technique and a high speed camera under the same conditions. Five different stages were studied and characterized. The final objective in this study was to visualize and identify fluid structures that occur during the deformation and breakup process in a continuously accelerated flow field in order to develop and fine tune physical-mathematical models.
\end{abstract}

\section{Keywords}

Droplet Breakup, Visualization, Accelerating Flow.

\section{Introduction}

Droplet aerobreakup has been studied mainly in shock tube [1,2] or wind tunnels facilities $[3,4,5]$, where the droplet suddenly experiences a high constant air speed. Two comprehensive reviews on this field are those of Guildenbecher et al. [6] and Theofanous [7]. In the first one, attention was paid to the breakup morphology and a similar classification of breakup mode as the one found in Pilch and Erdman [8] was established: Vibrational breakup, Bag Breakup, Multimode (Bag and Stamen), Sheet Stripping (Sheet Thinning), and Catastrophic Breakup. The second review made a good discussion on the two main mechanisms leading to breakup: RayleighTaylor piercing and shear-induced entrainment. However, these two reviews $[6,7]$ cover data obtained in facilities where droplets are suddenly exposed to a high constant velocity air stream. This is in contrast with the problem studied in this paper, where droplet are initially in a quiescent flow and then the air velocity starts to increase gradually with an acceleration that also increases, until the acceleration and the velocity reach values that allow for droplet breakup. The problem is a non-stationary problem and transient effects need to be considered. Accelerating and decelerated non-uniform flow fields have only been studied for non-deformable spheres $[9,10]$, or droplets that are small enough to neglect deformation [11] and it has proved to be a very complex problem since there still exist contratdicting results. Therefore, one of the novelties of this work is to study the aerobreakup of droplets in a non-stationary flow.

In order to visualize and identify fluid structures that occur during the water droplet deformation and breakup process, different techniques of image acquisition can be employed: shadowgraph images [3,4,12,13,14], interpherometry $[1,15]$ and direct illumination images [16]. Due to the difficulty associated to using direct illumination in transparent liquids, the shadowgraph technique has mainly been used in the past, providing only the distorted droplet contour. Previous studies by the authors were based on data obtained by shadowgraph illumination technique [17]. However, direct illumination allows for obtaining more information of the structure, which can provide a better understanding of the details of the process. This article compares visualization of the breakup of droplets in a non-stationary accelerating flow field using direct illumination technique to previous 
shadowgraph images. Only the bag and stamen breakup type is addressed. First the specific direct illumination technique is described, then the results are discussed and finally the main conclusions are summarized.

\section{Material and methods}

In order to achieve a continuously accelerated flow field the rotating arm facility at INTA was employed. The rotating arm facility had already been used to study water droplet deformation and breakup in a continuously accelerated flow field using the shadowgraph technique [17]. A photograph of the experimental setup can be observed in Figure 1. An airfoil model was attached to the end of a rotating arm whose length was $2.2 \mathrm{~m}$. At the same time, droplets of $0.8 \mathrm{~mm}$ of diameter were allowed to fall in the path of the incoming airfoil. These droplets were generated by means of a TSI MDG-100 droplet generator functioning on the bases of the Rayleigh theory. As the airfoil model approaches the droplets, the air velocity, as seen by the droplets, increases continuously. The flow field generated by the airfoil was characterized in advance using the Particle Image Velocimeter (PIV) technique. A complete description of this characterization can be found in [17]. This characterization allows us to correlate the conditions at which each breakup stage take place. The velocity flow field measurements, as seen from the airfoil reference system, can be observed in Figure 2. The airfoil model profile shape used is plotted in Figure 3. The airfoil model chord was $690 \mathrm{~mm}$, its leading edge radius was $103 \mathrm{~mm}$ and its span length was 200 $\mathrm{mm}$.

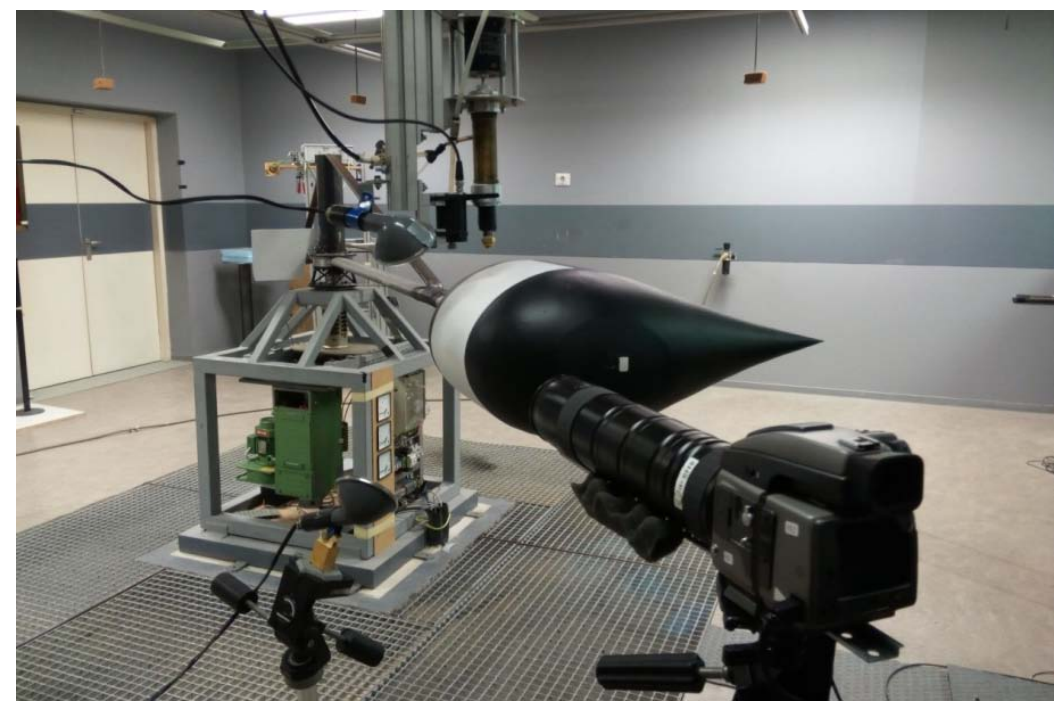

Figure 1. Photograph of the experimental setup.

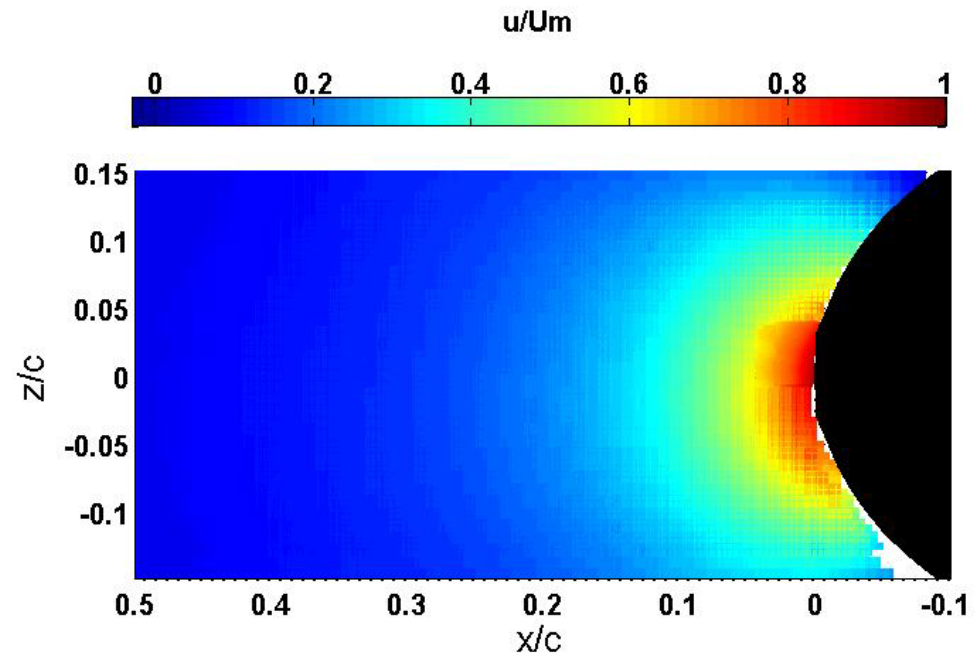

Figure 2. PIV measurements of the $2 \mathrm{D}$ vector magnitude of the velocity flow field as seen from the airfoil reference system. 


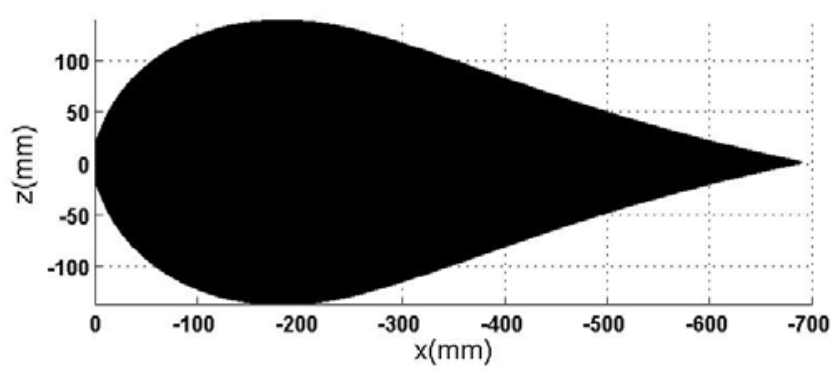

Figure 3. Airfoil model profile.

\section{Direct illumination technique}

The key element in the acquisition data technique presented in this paper is this illumination technique. The front part of the model was painted white to reflect the light and to increase the droplet illumination. The advantage of using the model as a light reflector is that the model is very close to the droplets when they breakup providing the maximum amount of light. Two flashes lamps, the 1538-A Strobotac Electronic Stroboscopic and the1539-A Stroboslave, illuminate the droplets and the airfoil model when it approaches the droplets (See Figure 4). Each flash lasted on the order of $3 \mu \mathrm{s}$. A diode photodetector trigger was installed at a certain position of the model path. The flashes are delayed a certain time from the trigger signal using a Programmable Timing Control Hub IDT. By choosing the delay time, the distance of the model to the droplets is varied. This allows for taking images at different stages of the deformation and breakup process. Photographs are taken by the high resolution camera Hasselblad H3DII-39, which has a resolution of 7216 pixels (width) $\times 5412$ pixels (height). The magnification was $145 \mathrm{pix} / \mathrm{mm}$. The lens used were: HC 4/200 mm, extensions H52 mm, H26 mm, H13 mm and a converter H1.7x. The camera setup was ISO-200 and f/6.8.

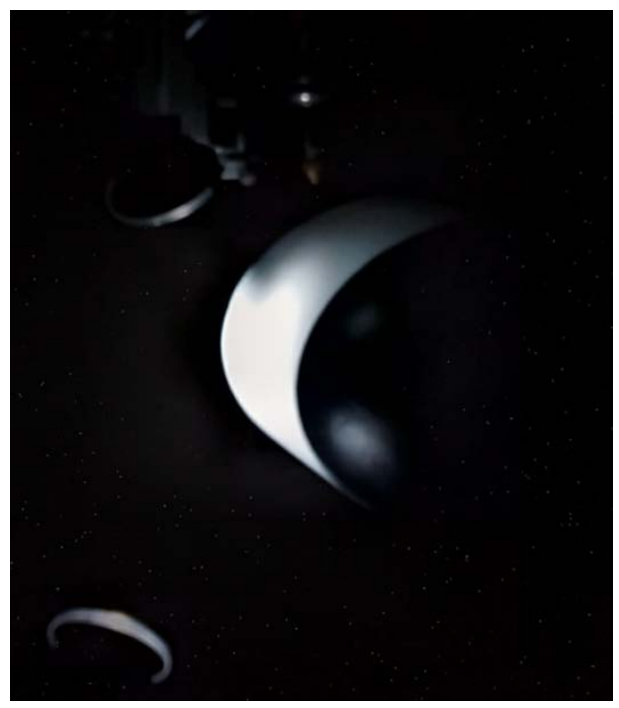

Figure 4. New illumination technique

An example of the images recorded by this illumination technique can be observed in Figure 5 . The airfoil model was moving at $80 \mathrm{~m} / \mathrm{s}$ and the initial droplets diameter was $0.8 \mathrm{~mm}$. 


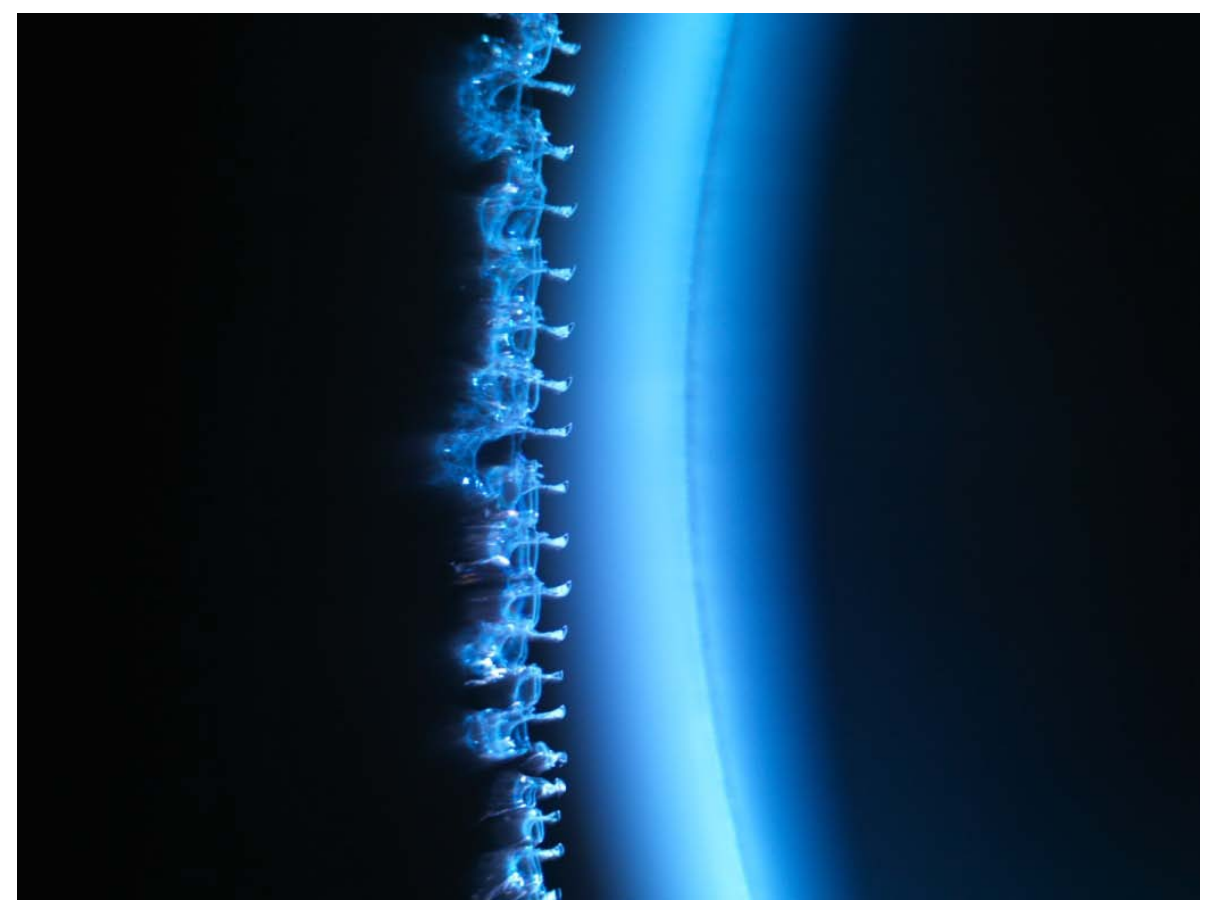

Figure 5. An example of the images taken by the direct illumination technique.

\section{Shadowgraph illumination technique}

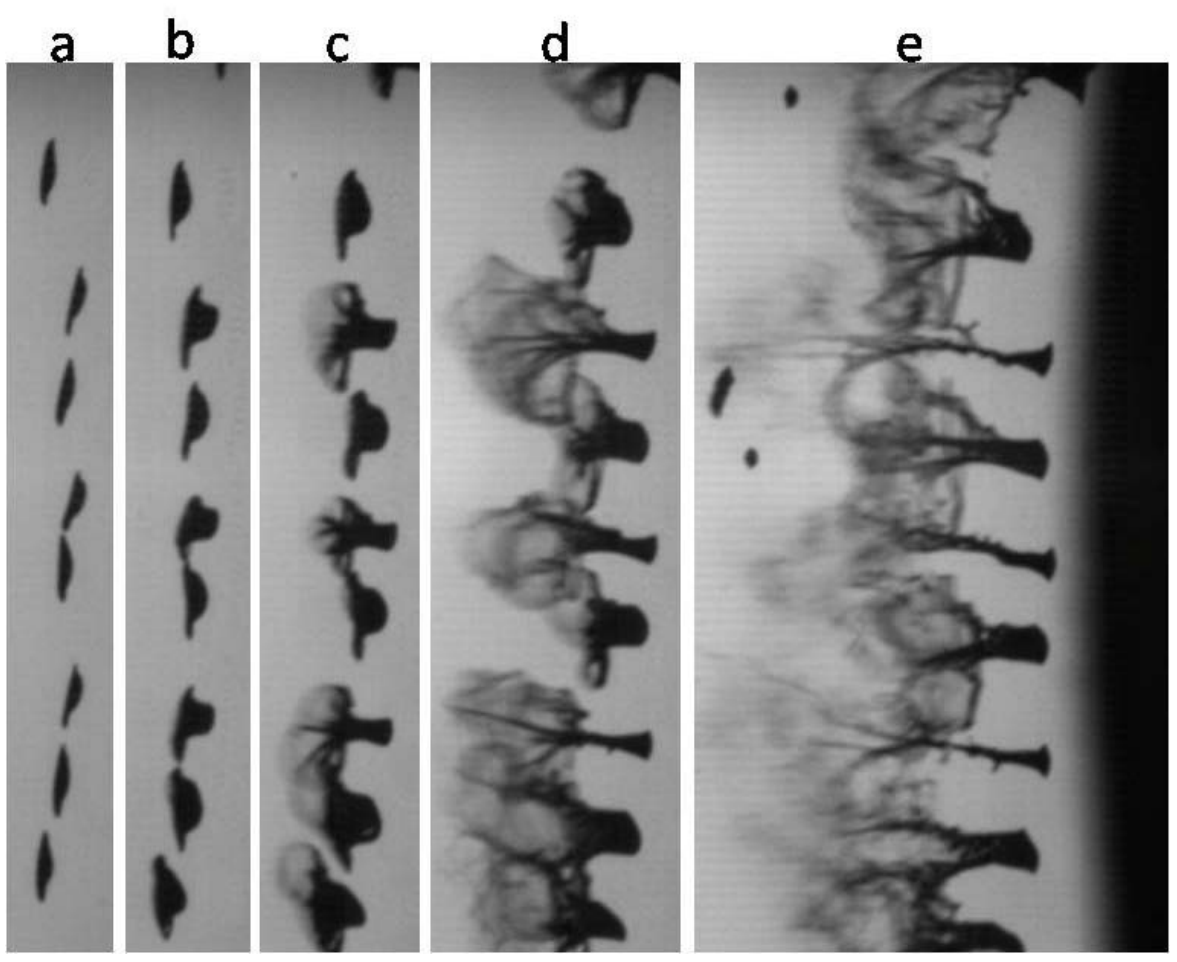

Figure 6. Five shadowgraph images that corresponds to the five stages found: a) Oblate spheroid shape (distance to the model $22 \mathrm{~mm}$, air velocity $59 \mathrm{~m} / \mathrm{s}$ ); b) Hat-like shape (distance to the model $16 \mathrm{~mm}$, air velocity $64 \mathrm{~m} / \mathrm{s}$ ); c) Mushroom-like shape (distance to the model $10 \mathrm{~mm}$, air velocity $69 \mathrm{~m} / \mathrm{s}$ ); d) Parachute-like shape (distance to the model $6 \mathrm{~mm}$, air velocity $74 \mathrm{~m} / \mathrm{s}$ ) and e) Fountain-like shape (air velocity $79 \mathrm{~m} / \mathrm{s}$ ).

In order to compare the photographs taken using direct illumination technique to the traditional shadowgraph technique, additional tests using shadowgraph illumination were also employed. Instead of a high resolution 
camera, a high speed camera at $75000 \mathrm{fps}$ was used with a resolution of $192 \times 312$ pixels. A xenon lamp of 2000 W illuminated back from the droplet background. The magnification was $14.26 \mathrm{pix} / \mathrm{mm}$. The advantage of using a high speed video camera was that a single droplet can be tracked, while the higher resolution camera was only able to take a single shot (photo camera). Either of the two ways may be helpful in faster understanding. An image of the evolution of deformation and breakup process can be observed in Figure 6.

\section{Results and discussion}

A sequence of the images as the droplets approach the model using both direct illumination technique and shadowgraph illumination are shown in Figures 6 and 7 respectively. It can be observed that, despite of the differences in the incoming flow field topology, droplets undergo a similar process to that found in the literature known as "bag and stamen" or "multimode" breakup mode. The breakup process has been divided into five stages summarized in Table 1, where characteristic distances from the leading edge of the droplet to the leading edge of the model at the centerline and their corresponding air velocities are given. Additionally Reynolds numbers and Weber numbers based on the droplet diameter and the instantaneous air velocity are also provided in Table 1.

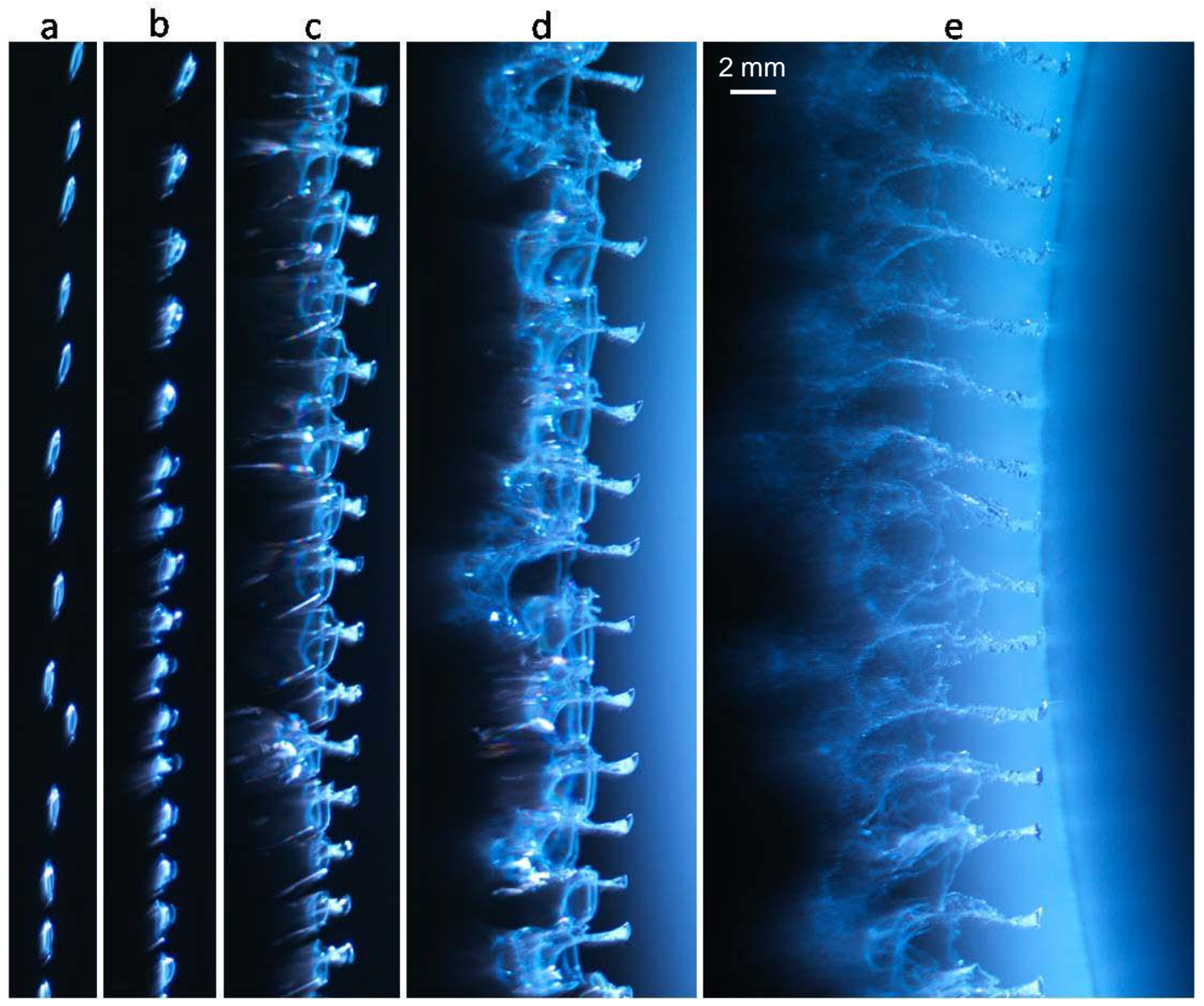

Figure 7. Five cropped images that correspond to the five stages found: a) Oblate spheroid shape (distance to the model 22 $\mathrm{mm}$ ); b) Hat-like shape (distance to the model $16 \mathrm{~mm}$ ); c) Mushroom-like shape (distance to the model $10 \mathrm{~mm}$ ); d) Parachutelike shape (distance to the model $5 \mathrm{~mm}$ ) and e) Fountain-like shape.

Table 1. Breakup stages found.

\begin{tabular}{c|c|c|c|c|c}
\hline \hline $\begin{array}{c}\text { Breakup } \\
\text { Stage }\end{array}$ & $\begin{array}{c}\text { Droplet Contour Shape } \\
\text { (observed in shadowgraph image) }\end{array}$ & $\begin{array}{c}\text { Distance to the } \\
\text { model }(\mathrm{mm})\end{array}$ & $\begin{array}{c}\text { Air velocity } \\
(\mathrm{m} / \mathrm{s})\end{array}$ & $\mathrm{Re}$ & We \\
\hline \hline a & Oblate Spheroid & 20 & 60 & 3230 & 48 \\
b & Hat-like shape & 15 & 65 & 3450 & 55 \\
c & Mushroom-like shape & 10 & 69 & 3700 & 63 \\
d & Parachute-like shape & 5 & 74 & 3970 & 73 \\
e & Fountain-like shape & 0 & 80 & 4270 & 84 \\
\hline \hline
\end{tabular}


Droplets that are initially spherical undergo a deformation process where they come to resemble an oblate spheroid due to the higher pressures found in the front and rear sides of the droplet and the lower pressures zone in the droplet rim. During this process, the maximum diameter of this oblate spheroid increases while the minimum diameter decreases. Then, there is an instant where the minimum diameter no longer decreases, but starts to increase. Theofanous et al [7] argued that this is due possibly to the Rayleigh-Taylor piercing at the forward side of the droplet. Figure 8 shows an image at this point. It can be observed the onset of a bulge starting on the right side, while an incipient recess in the left side is also evidenced thanks to the direct illumination technique. The incipient recess in the left side was not considered previously in the theoretical modelling [18]. Additionally the ratio of the bulge height to the droplet maximum diameter observed is greater than the typical multimode or bag and stamen morphology [19]. Then this bulge evolves towards to a hat cup that increases in length. A possible physical explanation for the increase in length of the hat cup is that the mass of the bulge is greater than the mass of the brim, which leads to a greater inertia.

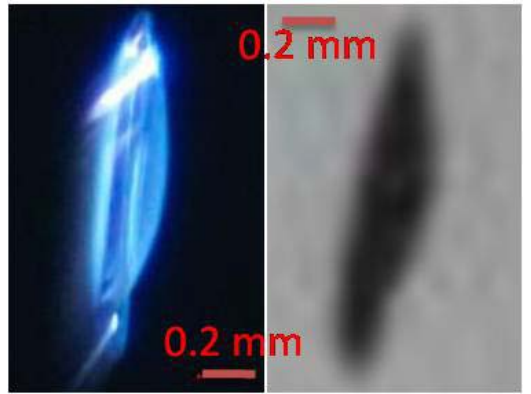

Figure 8. Oblate Spheroid comparison. Distance from the leading edge of the droplet to the model of $20 \mathrm{~mm}$.

Figure 9 shows the next stage, where a hat-like shape is evidenced. It can be observed the brim of the hat, where a concavity seems to be discerned.

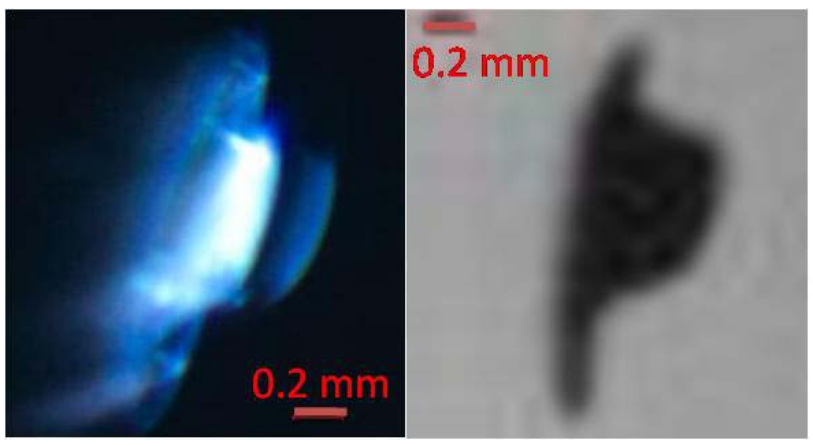

Figure 9. Hat-like shape comparison. Distance from the leading edge of the droplet to the model of $15 \mathrm{~mm}$.

In the next image, in Figure 10, the initial concavity in the brim of the hat has grown considerably by the time the traditionally mushroom-like shape is observed in the shadowgraph image. It can be observed that the rear side of the droplet (the left-side in the picture), has a hole in the middle whose length could even be guessed by the different colour observed in the image. The diameter of the brim has also increased being now of the order of 2.2 $\mathrm{mm}$. The depth of the concavity of the brim was measured to be of the order of $0.75 \mathrm{~mm}$. This direct illumination technique provides new information on that very feature at this point. From the shadowgraph image, it could be concluded that the rear part of the droplet was a bag; however from the new images it is evident the appearance of the central hole. The same is inferred from the parachute stage in Figure 10 also: although it could be inferred that there exists a half spherical big bag acting as a parachute, a toroidal bag with a central rear hole is evidenced actually in the new figure. Further studies are needed to explain from a physical point of view the new features found. 

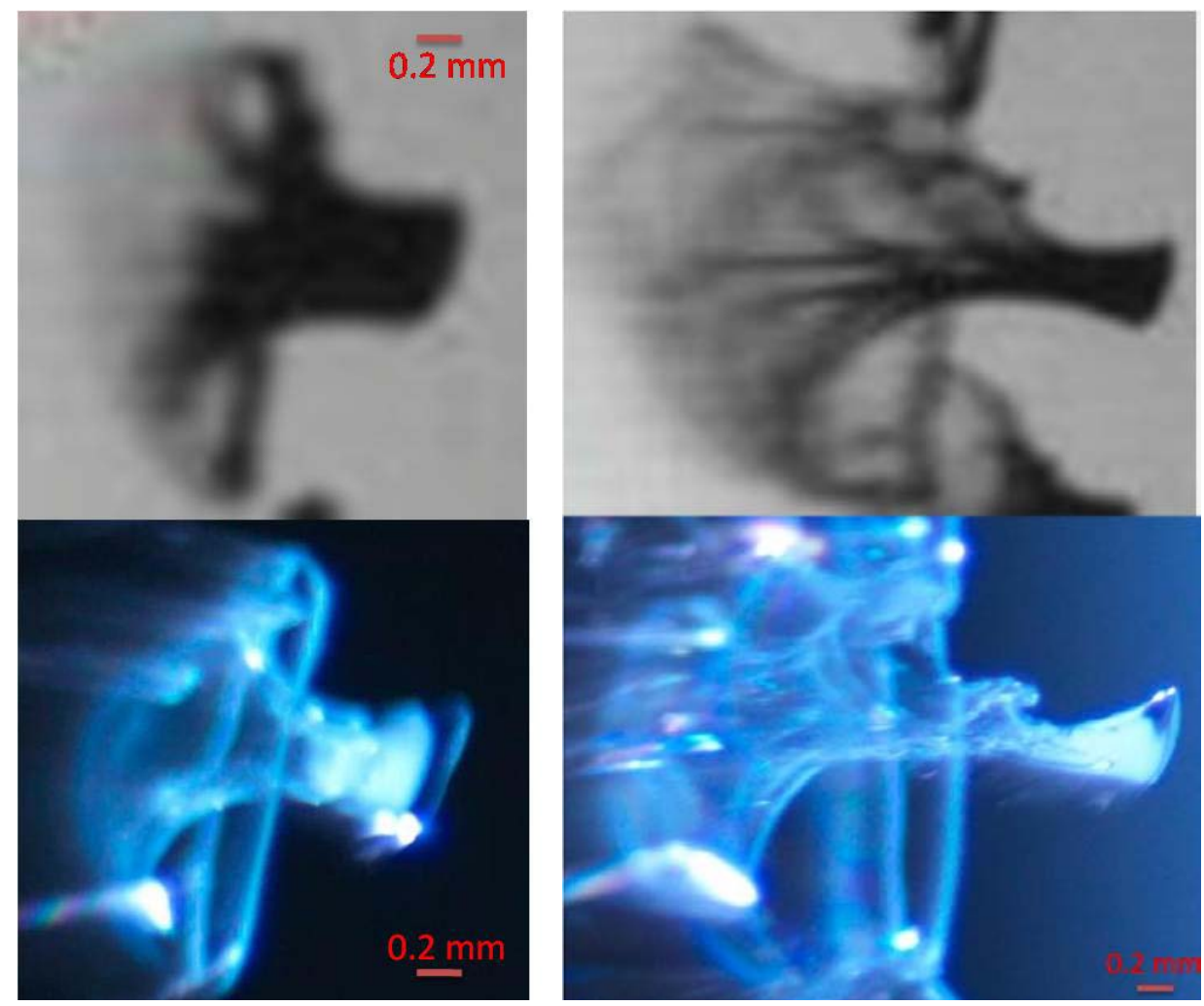

Figure 10. On the left: Mushroom-like shape comparison; distance from the leading edge of the droplet to the model of $10 \mathrm{~mm}$. the right: Parachute-like shape comparison; distance from the leading edge of the droplet to the model of $5 \mathrm{~mm}$.

Finally, the toroidal bag breaks and a fountain-like shape appear. Then the stamen, which was the tube that connected the toroidal bag to the rest of the droplet starts to break up as well. Figure 11 shows this last stage. It seems that most of the remaining droplet mass is located in the right hand side of the stamen.

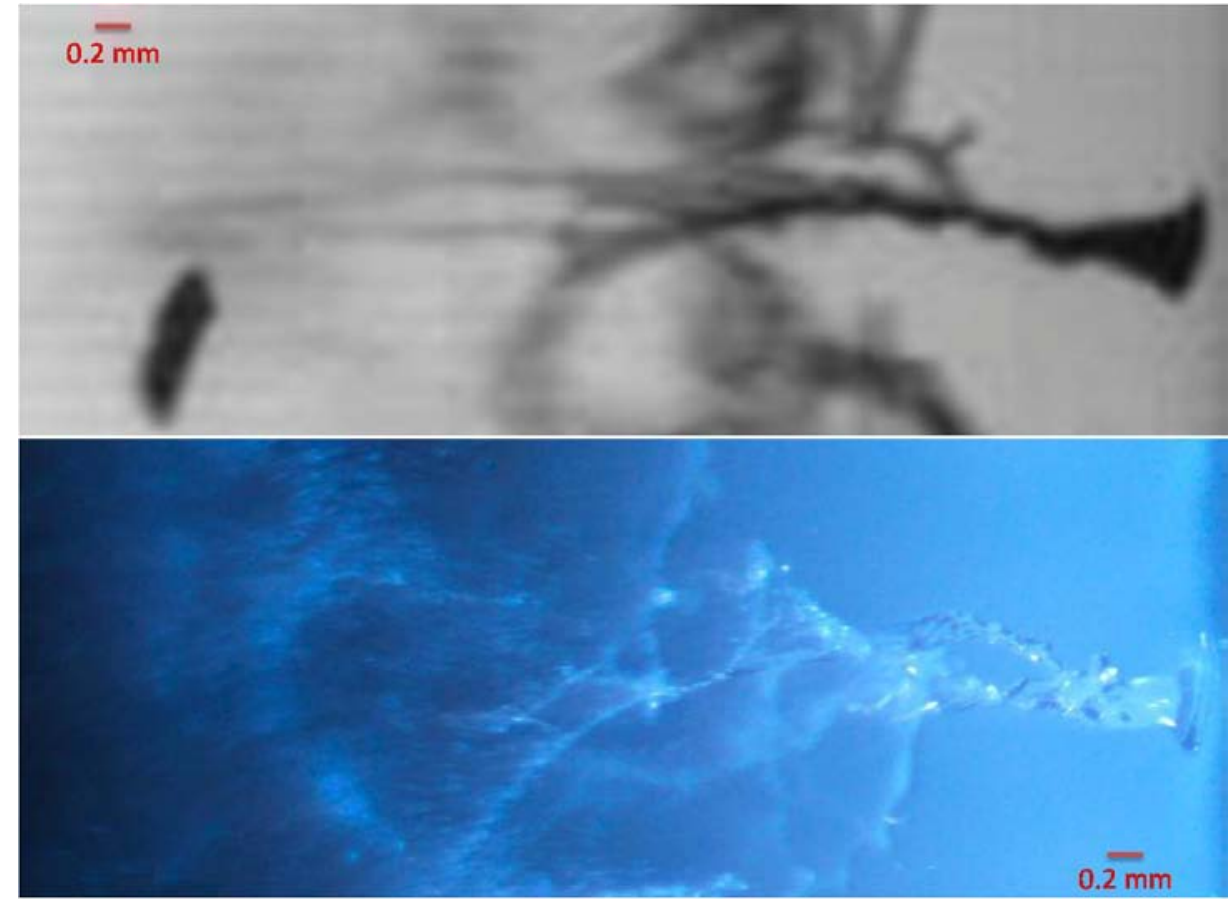

Figure 11. Fountain-like shape comparison. 


\section{Conclusions}

The deformation and breakup process of $0.8 \mathrm{~mm}$ diameter droplets subjected to a continously accelerating flow field has been studied by means of a new direct illuminaiton technique. The topology of the breakup was the type of "bag and stamen" or "multimode" breakup. Five different stages in the process were identified and characterized: a) Oblate spheroid shape, b) Hat-like shape, c) Mushroom-like shape, d) Parachute-like shape and e) Fountain-like shape. Direct illumination images obtained provide additional information regarding fluid structures that occur during the deformation and breakup process. Out from the beginning of the breakup process, a hole appears in the rear part of the droplet that was not inferred from previous observations. This hole is increasing in size as the stamen increases until the stamen finally breaks up. Further studies are needed to explain from a physical point of view the new features found.

Though the direct illumination technique has proved to be a good approach in this case, some problems have also been encountered. Very high light intensity was needed mainly due to two reasons: the short duration times of the phenomena (which led to the necessity of short flash duration) and the low reflection of the droplet due to its transparency. In order to obtain more light intensity, the reflector (the incoming airfoil model painted in white) could be placed very close to the droplet, thereby allowing for a larger amount of light. Due the direct illumination used, glints also appear in the photographs.

\section{Acknowledgements}

A. Velazquez was funded by the Spanish Ministry of Economy and Competitivenes (Ministerio de Economia y Competitividad) under research contract DPI2016-75296-P and gratefully acknowledges this support. A. GarcíaMagariño was funded by Spanish Ministry of Defense under the INTA program "Termofluidodinámica".

\section{Nomenclature}

C chord [mm]

u velocity $\left[\mathrm{m} \mathrm{s}^{-1}\right]$

$\mathrm{U}_{\mathrm{m}} \quad$ model velocity $\left[\mathrm{m} \mathrm{s}^{-1}\right]$

$\mathrm{x} \quad$ horizontal distance $[\mathrm{mm}]$

z vertical distance $[\mathrm{mm}]$

\section{References}

[1] Wierzba, A., Takayama, K., 1988, AIAA Journal, 26(11), pp. 1329-1331

[2] Hsiang, L.P., and Faeth, G. M., 1992, International Journal of Multiphase Flow, 18(5), pp. 635-652.

[3] Luxford, G., Hammond, W., and Ivey, P., January 2005, 43rd Aerospace Sciences Meeting and Exhibit.

[4] Wierzba, A., 1990, Experiments in Fluids, 9, pp. 59-64.

[5] Theofanous, T. G., and Li, G.J., 2008, Physics of Fluids, 20, 052103 pp:1-14

[6] Guildenbecher, D. R., Lopez-Rivera, C., and Sojka, P. E., 2009, Experiments in Fluids, 46(3), pp. $371-402$.

[7] Theofanous, T. G., 2011, Annual Review of Fluid Mechanics, 43, pp. 661-690

[8] Pilch, M., and Erdman, C. A., 1987, International Journal of Multiphase Flow, 13(6), pp. 741-757.

[9] Journal G., Houas L., Igra, O., Estivalez, J.-L., Devals, C., Meshkow, E. E., 2007, Proceeding of Real Society A, 463, pp. 3323-3345.

[10] Igra, O., Takayama, K., 1993, Proceeding of Real Society A, 442, pp. 231-247.

[11] Temkin, S., Metha, H. K., 1982, Journal of Fluid Mechanics, 116, pp. 297-312.

[12] Theofanous, T. G., Li, G.J., and Dinh, T. N., 2004, Transactions of the ASME, 126, pp: 516-527.

[13] Kwon, K.-S., Kim, H.-S., and Choi, M., 2016, Review of Scientificic Instruments, 87, 035101 pp: 1-10.

[14] Gobyzov, O., Lozhkin, Y., Ryabov, M. and Markovich, D., 2016, EPJ Web of Conferences, 114, 02031 pp: 16.

[15] Pavlov, I. N., Rinkevichyus, B. S., and Tolkachev, A. V., June 25-28, 2012, $15^{\text {th }}$ International Symposium on Flow Visualization, 114, 02031 pp: 1-6.

[16] Illias, S., Ishak, M. S. A., Hussain, S. and Ismail, K. A., 2016, International Journal of Applied Engineering Research, 11(22), pp: 10832-10837.

[17] Garcia-Magariño, A., Sor, S., and Velazquez, A. 2015, Aerospace Science and Technology, 45, pp. $190-500$.

[18] Wang, C., Chang, S., Wu, H., Ding, L., and Thompson, J. M. 2015, Atomization and Sprays, 25(10), pp. 857869.

[19] Theofanous, T. G., Li, G. J., and Dihn, T. N. 2004, Transactions of the ASME, 126, pp. 516-527. 